\title{
Interdigitating Dendritic Cell Sarcoma of the Leg
}

\author{
Zdenek Adam $^{\mathrm{a}}$ Luděk Pour ${ }^{\mathrm{a}} \quad$ Karel Veselý $^{\mathrm{b}} \quad$ Marta Krejčí $^{\mathrm{a}} \quad$ František Fakan $^{\mathrm{c}}$ \\ Ferdinand Hofstädter $^{\mathrm{d}}$ Tomáš Büchler ${ }^{\mathrm{e}}$ Roman Hajek $^{\mathrm{a}}$ Jiř́ Mayer ${ }^{\mathrm{a}}$ \\ ${ }^{a}$ Department of Internal Medicine - Hematooncology, Faculty of Medicine and University Hospital, \\ ${ }^{b}$ 1st Department of Pathology, Faculty of Medicine and St Anna University Hospital, Masaryk University, Brno, \\ ${ }^{c}$ Sikl Institute of Pathology, Faculty of Medicine and University Hospital, PIzen, Czech Republic \\ ${ }^{\mathrm{d}}$ Institute for Pathology, Faculty of Medicine, Regensburg University, Germany \\ ${ }^{e}$ Department of Oncology, Thomayer University Hospital and 1st Faculty of Medicine, Charles University, Prague, Czech Republic
}

Interdigitating dendritic cell sarcoma is a very rare malignancy. In the few cases reported in the literature, the primary tumor occurred in the peripheral lymph nodes, spleen, lungs, bladder, duodenum, testis, or breast [1]. Surgery was the main treatment modality used in the previously reported cases. Only 1 case study described a response to ABVD chemotherapy (doxorubicin, bleomycin, vinblastine, dacarbazine) [2] Thus, there are few data on the chemosensitivity of interdigitating dendritic cell sarcoma. Somewhat more information is available on the systemic treatment of a related type of sarcoma composed of malignant follicular dendritic cells. However, only limited responses have been achieved in follicular dendritic cell sarcoma using combination chemotherapy with regimens including $\mathrm{CHOP}$ (cyclophosphamide, doxorubicin, vincristine, prednisone), ABVD, DHAP (dexamethasone, cytarabine, cisplatin), EPOCH (etoposide, vincristine, doxorubicin, cyclophosphamide, prednisone), ICE (ifosfamide, carboplatin, etoposide), or cisplatin/epirubicin [1,3]. Only 1 report describes complete response after imatinib in combination with other drugs [4]. There are no published data on the efficacy of high-dose chemotherapy for interdigitating dendritic cell sarcoma.

We report on a 26-year-old male patient who was diagnosed with interdigitating dendritic cell sarcoma in the proximal part of the tibia. The tumor involved the proximal epiphysis of the tibia and had invaded the adjacent soft tissues. The diagnosis was made based on excision biopsy, and the histology was confirmed by 3 independent histopathologists in the Czech Republic and Germany. Staging was performed using positron emission tomography (PET) which showed a pathologic uptake of glucose in the primary lesion with a standardized uptake value (SUV) of 7.71, and in an ipsilateral inguinal lymph node with an SUV of 4.28. Pelvic and abdominal lymph nodes were not affected. A computed tomography (CT) scan confirmed inguinal lymphadenopathy but did not reveal any additional lesions. The suspect inguinal lymph node was extirpated, and sarcoma infiltration was confirmed by histological examination. Because of the extent of the disease, we decided not to proceed with radical surgery but to treat with systemic chemotherapy. As there is a paucity of data on the chemosensitivity of these tumors, we chose the MegaCHOP protocol used for aggressive lymphomas: cyclophosphamide $2,000 \mathrm{mg} / \mathrm{m}^{2}$, doxorubicin $50 \mathrm{mg} / \mathrm{m}^{2}$, vincristine $1.4 \mathrm{mg} / \mathrm{m}^{2}$ on day 1 , and prednisone $100 \mathrm{mg}$ daily on days $1-5$. Granulocyte colony-stimulating factor (G-CSF) was applied from day 7. The interval between cycles was 21 days. However, there was no regression of the tumor after 4 cycles of this chemotherapy, and we switched to ESHAP chemotherapy (cisplatin $25 \mathrm{mg} / \mathrm{m}^{2}$ on days $1-4$, etoposide $60 \mathrm{mg} / \mathrm{m}^{2}$ on days $1-4$, cytarabin on day 4) followed by peripheral hematopoietic stem cell harvest. One month after successful stem cell mobilization, we proceeded to high-dose chemotherapy with the BEAM regimen (BCNU $310 \mathrm{mg} / \mathrm{m}^{2}$ on day -7 , cytarabin $120 \mathrm{mg} / \mathrm{m}^{2}$ twice daily on days -6 to -3 , etoposide $240 \mathrm{mg} / \mathrm{m}^{2}$ on days -6 to -3 , and melphalan $140 \mathrm{mg} / \mathrm{m}^{2}$ on day -2 . High-dose chemotherapy and autologous hematopoietic stem cell transplantation were performed without complications.

Several days before the start of high-dose chemotherapy, another PET scan showed the persistence of the malignant lesion in the proximal tibia with an SUV of 4.6. No further foci of pathologic glucose uptake were demonstrated. Because of these findings, after the patient recovered from the autologous transplant, he went on to receive radiotherapy to the proximal tibia and distal femur at a dose of 70 Gy given in 35 fractions from 2 latero-lateral fields with a decrease in safety margins after the dose of $50 \mathrm{~Gy}$. Three months after the completion

\section{KARGER}

Fax +49 7614520714

Information@Karger.de

www.karger.com (c) 2009 S. Karger GmbH, Freiburg

Accessible online at:

www.karger.com/onk
Prof. Dr. Zdenek Adam

University Hospital Brno

Jihlavská 2062500 Brno

Czech Republic

Tel. +420 54719-3644, Fax -3603

z.adam@fnbrno.cz 
of radiotherapy, a third PET scan was done revealing persistent activity in the site of the primary tumor (SUV 2.69) and reappearance of the pathologic activity in the inguinal region (SUV 4.09). Inguinal lymph nodes were widely dissected and removed, and histological examination confirmed a dendritic cell sarcoma recurrence. The treatment continued with radiotherapy to the inguinal region. Six months after the inguinal surgery and irradiation, the patient presented with a palpable lower abdominal mass, and CT scan demonstrated massive iliac lymphadenopathy which was also positive on PET scan (SUV 5.9-6.73). At present, the patient is undergoing palliative chemotherapy with 2-chlorodeoxyadenosine.

To our knowledge, this is the first report of interdigitating dendritic cell sarcoma with primary lesions in the bone and adjacent soft tissues. The tumor was resistant to both conven- tional lymphoma-type chemotherapy and high-dose chemotherapy. Surgery remains the only option with curative potential in this rare type of malignancy if radical removal of the tumor is feasible.

\section{Acknowledgement}

This study was supported in part by grants VZ MSM 0021622434 from the Czech Ministry of Education and LC06027 from the Masaryk University, Brno, Czech Republic.

\section{Conflict of Interest}

The authors declare that they have no conflict of interests.

\section{References}

1 Kairouz S, Hashash J, Kabbara W, McHayleh W, Tabbara IA: Dendritic cell neoplasms: an overview. Am J Hematol 2007;82:924-928.

$\checkmark 2$ Olnes MJ, Nicol T, Duncan M: Interdigitating dendritic cell sarcoma: a rare malignancy responsive to ABVD chemotherapy. Leuk Lymphoma 2002;43: 817-821.
De Pas T, Spitaleri G, Pruneri G, Curigliano G, Noberasco C, Luini A, Andreoni B, Testori A, de Braud F: Dendritic cell sarcoma: an analytic overview of the literature and presentation of original five cases. Crit Rev Oncol Hematol 2008;65:1-7.
Azim HA, Elsedewy E, Azim HA Jr: Imatinib in the treatment of follicular dendritic sarcoma: a case report and review of literature. Onkologie 2007;30: 381-384. 